\title{
Structured jets in TeV BL Lac objects and radiogalaxies
}

\section{Implications for the observed properties}

\author{
G. Ghisellini ${ }^{1}$, F. Tavecchio ${ }^{1}$, and M. Chiaberge ${ }^{2}$ \\ 1 INAF - Osservatorio Astronomico di Brera, via Bianchi 46, 23807 Merate, Italy \\ e-mail: gabriele@merate.mi .astro.it \\ 2 IRA/CNR, via Gobetti 101, 40129, Bologna, Italy
}

Received 3 June 2004 / Accepted 28 October 2004

\begin{abstract}
TeV BL Lacertae objects require extreme relativistic bulk motions in the gamma-ray emission region, but at the VLBI scale their radio knots hardly move. The same sources show evidence, in radio, of a structure made of a fast spine plus a slow layer. We propose that this structure exists even on the spatial scale of regions responsible for the gamma-ray emission. One component sees the (beamed) radiation produced by the other, and this enhances the inverse Compton emission of both components. In addition, this allows the magnetic field to be nearly in equipartition with the emitting particles. The inverse Compton emission of the spine is anisotropic in its frame, possibly producing a deceleration of the spine by the Compton rocket effect. In this scenario, the slow layer is also a relatively strong high-energy emitter, and thus radiogalaxies become potentially detectable by GLAST.
\end{abstract}

Key words. galaxies: jets - galaxies: BL Lacertae objects: general - radio continuum: galaxies radiation mechanisms: non-thermal - gamma-rays: theory

\section{Introduction}

There is growing evidence from VLBI studies that pc-scale jets in strong TeV BL Lacs move slowly (Edwards \& Piner 2002; Piner \& Edwards 2004; Giroletti et al. 2004). On the contrary, the bright and rapidly variable $\mathrm{TeV}$ emission implies that at the jet scales where this emission originates, the jet should be highly relativistic. This is necessary in order to avoid the absorption of $\mathrm{TeV}$ photons by the IR radiation produced cospatially to the $\mathrm{TeV}$ emission (see e.g. Dondi \& Ghisellini 1995). Furthermore, fitting the SED of TeV sources with a simple, one-zone homogeneous synchrotron self-Compton (SSC) model makes it possible to determine a unique set of physical parameters of the emitting region (Tavecchio et al. 1998), and in fact all authors that apply SSC models derive similar values of the Doppler factor, in the range 10-20, when the TeV spectrum is not de-reddened by the absorption of $\mathrm{TeV}$ photons by the IR background (Tavecchio et al. 2001; Kino et al. 2002; Ghisellini et al. 2002; Katarzynski et al. 2003), and larger (up to 50) when the $\mathrm{TeV}$ spectrum is de-reddened (Krawczynski et al. 2002; Konopelko et al. 2003). It is therefore clear that the jet must suffer severe deceleration from the $\gamma$-ray emitting zone ( $\sim 0.1 \mathrm{pc}$ from the jet apex) to the VLBI $(\sim 1 \mathrm{pc})$ scale.

Prompted by this observational evidence, Georganopoulos \& Kazanas (2003, hereafter GK03) proposed that if the entire jet is rapidly decelerating in the $\gamma$-ray zone, then the base of the jet, still moving fast, will see the radiation produced at the end of the deceleration zone relativistically boosted. This "extra" radiation will favor inverse Compton emission, making it possible to derive less extreme values for the physical parameters with respect to a pure one-zone SSC model. Our paper is germane to the one of GK03, but we study the alternative hypothesis that the jet is structured not in the radial, but in the transverse direction, being composed by a slow layer and a fast spine.

We are motivated by the recent observational evidence coming from detailed VLBI (including space VSOP observations) radio maps, showing, in Mkn 501, a limb brightening morphology, interpreted as evidence of a slower external flow surrounding a faster spine (Giroletti et al. 2004). Similar results have been obtained for a few radiogalaxies (Swain et al. 1998; Owen et al. 1989; Giovannini et al. 1999). Apart from observational evidence, a spine-layer configuration for the jet has been proposed in the past on the basis of theoretical arguments (e.g., Henri \& Pelletier 1991). In addition, the existence of a velocity structure has also been suggested to explain some observed properties of radiogalaxies, such as their magnetic field configuration (Komissarov 1990; Laing 1993), and to overcome problems in unifying radiogalaxies with BL Lac objects (Chiaberge et al. 2000).

We also hope that this assumption helps to find a possible reason for the deceleration of (at least a part of) the jet, which was postulated by GK03. A slow layer could in fact be the result of the interaction of the "walls" of the jet with the ambient medium, or simply of a jet acceleration which is 
a function of the angular distance from the jet axis ${ }^{1}$, producing a velocity structure. Kelvin-Helmholtz instabilities (for a review see Ferrari 1998), while important for the formation of the layer, may not decelerate (and destroy) the entire jet (e.g. Bodo et al. 2003), especially if the jet itself is not continuous, but "intermittent", as in the internal shock scenario (Ghisellini 1999; Spada et al. 2001; Guetta et al. 2004). Note in fact that the dynamical timescale involved in internal shocks is approximately the light crossing time across the source, while instabilities can grow with the sound speed.

As in the decelerating scenario proposed by GK03, there will be a strong radiative interplay and feedback between the layer and the spine: each part sees extra seed photons coming from the other part, and this will enhance the inverse Compton emission of both components. This may help to explain why radiogalaxies can also be relatively strong $\gamma$-ray emitters, as suggested by the Comptel and EGRET (onboard the Compton Gamma Ray Observatory) detection of Centaurus A (Steinle et al. 1998, and references therein), the recent identifications of NGC 6251 with an EGRET source (Mukherjee et al. 2002), and the possible detection of M 87 at TeV energies (Aharonian et al. 2003). This emission, coming from the inner part of the relativistic jet of radiogalaxies, should be characterized by a pronounced variability: this could help to distinguish it from the high energy radiation coming from the more extended (kpc) parts of the jet, as suggested by Stawarz et al. (2003).

In Sect. 2 we present the basic assumptions of the model and in Sect. 3 we discuss what we think is a major outcome of the scenario we are proposing, namely the possibility that the spine can recoil under the effect of its own inverse Compton emission (Compton rocket effect). We apply the model in Sect. 4 to Mkn 421, Mkn 501, Cen A and also to NGC 6251, assumed to be "paired" with the classic BL Lac PKS 0735+178. Even if the presented "fits" 2 are not unique solutions (given the large number of free parameters), they illustrate the radiative feedback between the spine and the layer. Since it is natural, in our model, that the inverse Compton emission of the layer is also enhanced, we stress in Sect. 5 that radiogalaxies, not only blazars, can be bright $\gamma$-ray emitters, and we present a preliminary list of possible candidates for detection by GLAST. In Sect. 6 we discuss our results and draw our conclusions.

\section{The model}

We assume that the layer can be approximated as a hollow cylinder, with external radius $R_{2}$, internal radius $R$ and width $\Delta R_{1}{ }^{\prime \prime}$, as measured in the comoving frame of the layer ${ }^{3}$. The comoving volume of the layer is then $V_{1}^{\prime \prime}=\pi\left(R_{2}^{2}-R^{2}\right) \Delta R_{1}^{\prime \prime}$. For the spine we also assume a cylindrical geometry, with the same $R$ and a width $\Delta R_{\mathrm{s}}^{\prime}$, as measured in the comoving frame of the spine. The active volume of the spine is then $V_{\mathrm{s}}^{\prime}=\pi R^{2} \Delta R_{\mathrm{s}}^{\prime}$.

\footnotetext{
${ }^{1}$ Note that the possibility of structured jets has been proposed and somewhat explored also for Gamma-ray Bursts, see Rossi et al. (2001); Zhang \& Meszaros (2002).

2 What we call "fit" is really a comparison of the model against the observational data.

3 Primed quantities are in the rest frame of the spine, double primed quantities are in the frame of the layer.
}

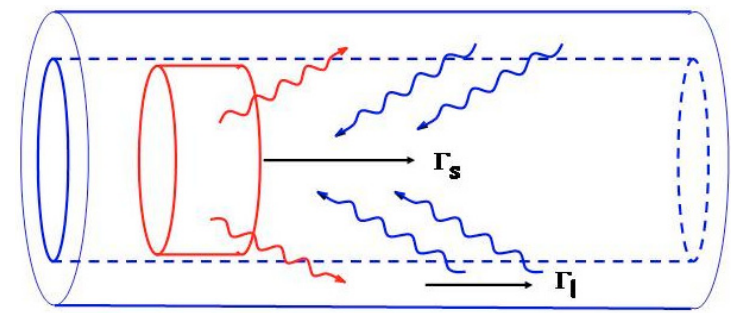

Fig. 1. Cartoon illustrating the layer+spine system.

Figure 1 illustrates the assumed geometry. The Lorentz factors of the spine and of the layer are $\Gamma_{\mathrm{s}}$ and $\Gamma_{\mathrm{l}}$, respectively, with $c \beta_{\mathrm{s}}$ and $c \beta_{1}$ the corresponding velocities. Since the spine and the layer move with different Lorentz factors, the radiation emitted by the spine (layer) is seen boosted by the layer (spine). With respect to a comoving observer at the same distance from the spine (layer), the radiation energy density is enhanced by a factor $\sim\left(\Gamma^{\prime}\right)^{2}$, with $\Gamma^{\prime}$ given by

$\Gamma^{\prime}=\Gamma_{\mathrm{s}} \Gamma_{\mathrm{l}}\left(1-\beta_{\mathrm{s}} \beta_{1}\right)$

Both structures emit by the synchrotron and the inverse Compton processes. The energy distribution of the emitting electrons, $N(\gamma)$, is assumed to extend down to $\gamma_{\min }$, and to have the shape:

$\begin{array}{llrl}N(\gamma)=K \gamma^{-n_{1}}\left[1+\left(\frac{\gamma}{\gamma_{b}}\right)^{n_{1}-n_{2}}\right] \mathrm{e}^{-\frac{\gamma}{\gamma_{\text {cut }}}} ; & \gamma>\gamma_{\text {min }} & \\ N(\gamma)=0, & \gamma \leq \gamma_{\text {min }} .\end{array}$

The normalization (i.e. $K$ ) of this distribution is found by imposing that $N(\gamma)$ produces a given intrinsic synchrotron luminosity, which is an input parameter of the model.

The seed photons relevant for the scattering process are produced not only by the spine (layer) electrons, but also by the layer (spine) ones. There is a strong feedback between the two components, which determines the amount of inverse Compton radiation emitted by both structures (if this process is important with respect to the synchrotron process). As a general rule, this feedback increases the inverse Compton flux, since both the spine and the layer see an enhanced radiation energy density. Since the ratio between the radiation and the magnetic energy densities $U_{\mathrm{B}} / U_{\text {rad }}$ measures the ratio between the inverse Compton and the synchrotron luminosities (even if, for scattering in the Klein Nishina regime, the two ratios are not equal), an enhanced $U_{\text {rad }}$ in turn implies a larger magnetic field. Therefore both the synchrotron and the inverse Compton luminosities can be produced by a reduced number of relativistic electrons (radiatively cooling in a shorter time with respect to the case of no feedback). This has important consequences for the energetics and the dynamics of the jet, and it will be discussed in Sect. 3, while in Sect. 4 we present Mkn 421 as an illustrative example.

The length of the layer, as observed in a frame comoving with the spine, is $R_{1}^{\prime}=R_{1}^{\prime \prime} / \Gamma^{\prime}$. Analogously, the length of the spine, as observed in the frame comoving with the layer, is $R_{\mathrm{S}}{ }^{\prime \prime}=R_{\mathrm{s}}^{\prime} / \Gamma^{\prime}$. In the following, we will always assume that the layer is longer than the spine, even in the frame of the spine. 
To calculate the radiation energy density of one component as observed by the other, we will assume the following:

- Let us call $\bar{R} \equiv\left(R_{2}+R\right) / 2$. In the comoving frame of the layer, the radiation energy density in the entire cylinder is assumed to be $U_{1}^{\prime \prime}=L_{1}^{\prime \prime} /\left(\pi \bar{R}^{2} c\right)$. In the frame of the spine, this radiation energy density is assumed to be boosted by a factor $\left(\Gamma^{\prime}\right)^{2}$, i.e. $U_{1}^{\prime}=\left(\Gamma^{\prime}\right)^{2} U_{1}^{\prime \prime}$.

- In the comoving frame of the spine, the radiation energy density within the spine is assumed to be $U_{\mathrm{s}}^{\prime}=L_{\mathrm{s}}^{\prime} /\left(\pi R^{2} c\right)$. In the frame of the layer, this radiation energy density is observed to be boosted by $\left(\Gamma^{\prime}\right)^{2}$, but also diluted (since the layer is larger than the spine) by a factor $\Delta R_{\mathrm{S}}{ }^{\prime \prime} / \Delta R_{\mathrm{l}}{ }^{\prime \prime}=$ $\left(\Delta R_{\mathrm{s}}^{\prime} / \Gamma^{\prime}\right) / \Delta R_{1}^{\prime \prime}$.

While the synchrotron and the SSC emission in the comoving frame are assumed to be isotropic, the inverse Compton process between electrons of the layer (spine) and seed photons produced by the spine (layer) is highly anisotropic. Dermer (1995) found the pattern of the emitted radiation from a moving blob immersed in a bath of seed photons (e.g. corresponding to the radiation produced by the broad line region of a powerful blazar), and pointed out the fact that in this case the external Compton radiation is more beamed than the synchrotron and SSC emission.

In our case the component contributing to the "external" radiation is not at rest with respect to the distant observer, but moves. To find the pattern of the emitted radiation it is convenient to move to the comoving frame of the emitter of the seed photons. Consider then the seed photons produced by the layer, and an observer comoving with the layer. In this frame, the spine is moving with $\Gamma^{\prime}$, and the photon frequencies produced by the spine are blueshifted by the Doppler factor ${ }^{4} \delta_{\mathrm{s}, 1}$. Going to the frame of the distant observer, these photons are further blueshifted by $\delta_{1}$. But the distant observer will see the same photons blueshifted by $\delta_{\mathrm{s}}$. This implies

$\delta_{\mathrm{s}, 1} \delta_{1}=\delta_{\mathrm{s}}$.

We can repeat the same argument for photons produced by the layer. We then have

$\delta_{\mathrm{s}, 1}=\frac{\delta_{\mathrm{s}}}{\delta_{\mathrm{l}}}=\frac{1}{\delta_{\mathrm{l}, \mathrm{s}}}$

This nice argument is due to GK03.

In the frame of the layer the external Compton radiation produced by the spine follows a pattern $\propto \delta_{\mathrm{s}, 1}^{4+2 \alpha}$ (Dermer 1995), where $\alpha$ is the spectral index of the emission $\left[F(v) \propto v^{-\alpha}\right]$, while the synchrotron and SSC emission follow the usual pattern $\propto \delta_{\mathrm{s}, 1}^{3+\alpha}$. If $I^{\prime}\left(v^{\prime}\right)$ is the monochromatic intrinsic intensity produced by the spine, we have

$I(v)=I^{\prime}\left(v^{\prime}\right) \delta_{\mathrm{s}, 1}^{4+2 \alpha} \delta_{1}^{3+\alpha}=I^{\prime}\left(v^{\prime}\right) \delta_{\mathrm{s}}^{3+\alpha}\left(\frac{\delta_{\mathrm{s}}}{\delta_{\mathrm{l}}}\right)^{1+\alpha}$

$I(v)=I^{\prime}\left(v^{\prime}\right) \delta_{\mathrm{s}}^{3+\alpha} \quad(\mathrm{S}, \mathrm{SSC})$.

${ }^{4} \delta=\Gamma^{-1}(1-\beta \cos \theta)^{-1}$, where $\theta$ is the viewing angle. Note that $\Gamma$ and $\theta$ are different in different frames. $\delta_{\mathrm{s}, \mathrm{l}}$ is defined as the beaming factor of the radiation produced in the spine as observed in the layer.

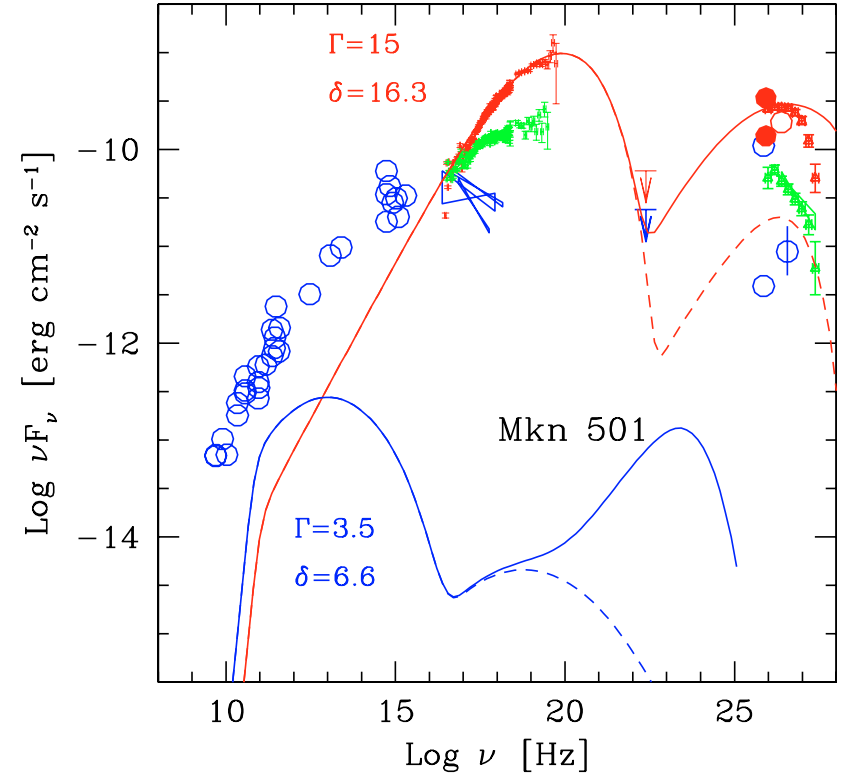

Fig. 2. Example of the SED produced by the spine-layer system, using the parameters listed in Table 1. Dashed lines correspond to the emission of the spine (layer) without taking into account the seed photons coming from the layer (spine). Data from Pian et al. (1998) and Djannati-Atai et al. (1999).

Here S stands for synchrotron, EC for "external Compton" (scattering of the seed photons coming from the layer). In the case of radiation produced by the layer, the transformation is the same, with $\delta_{\mathrm{s}}$ and $\delta_{1}$ interchanged.

Free parameters - For each component the input parameters are: $\Delta R^{\prime}, B, L_{\text {inj }}^{\prime}, \Gamma, \gamma_{\text {min }}, \gamma_{\mathrm{b}}, \gamma_{\text {cut }}, n_{1}, n_{2}$. Parameters equal for both components are $R$ and the viewing angle $\theta$. The outer radius of the layer $R_{2}$ must also be specified, but we will always use $R_{2}=1.2 R$. For $n_{2}>3, \gamma_{\text {cut }}$ becomes unimportant and we have a total of 18 parameters. While they appear (and are) many, we stress that the aim of this paper is to discuss the main effects of having the layer+spine structure, and not (yet) the exact determination of the physical quantities inside the source. In other words, we have to model two structures by observing the radiation coming from only one of them, and this will leave some ambiguity, unless we obtain information about the layer (spine) even if we are observing the spine (layer) emission ${ }^{5}$.

\section{Energetics and dynamics}

As we will see in the next section, the power spent by the jet to emit the inverse Compton radiation is significant, if compared with the total bulk kinetic power contained in the relativistic electrons and protons. As described above, this luminosity is emitted anisotropically in the comoving frame of the spine, hence the spine must recoil. Since this Compton rocket effect

${ }^{5}$ One possibility could be the observation, in radiogalaxies, of emission lines resulting from photoionization due to the spine radiation (Morganti et al. 1992), or the knowledge of the total power carried by the entire jet through estimates of the total energy and age of the extended radio structure (as done by Rawlings \& Saunders 1991). 
Table 1. Input parameters of the models for the layer and the spine shown in Figs. 2-5. Note that we have assumed that the viewing angles for PKS $0735+178$ and for NCG 6251 are $3^{\circ}$ and $20^{\circ}$ respectively.

\begin{tabular}{lllllllllllll}
\hline \hline & $\begin{array}{l}R \\
\mathrm{~cm}\end{array}$ & $\begin{array}{l}\Delta R^{\prime} \\
\mathrm{cm}\end{array}$ & $\begin{array}{l}L_{\text {syn }}^{\prime} \\
\mathrm{erg} \mathrm{s}^{-1}\end{array}$ & $\begin{array}{l}B \\
\mathrm{G}\end{array}$ & $\gamma_{\min }$ & $\gamma_{\mathrm{b}}$ & $\gamma_{\text {cut }}$ & $n_{1}$ & $n_{2}$ & $\Gamma$ & $\theta$ \\
\hline Mkn 501 (spine) & $3 \mathrm{e} 15$ & $1 \mathrm{e} 15$ & $2.5 \mathrm{e} 41$ & 1.3 & 1 & $3 \mathrm{e} 5$ & $1 \mathrm{e} 7$ & 1.7 & 2.6 & 15 & 3.5 \\
Mkn 501 (layer) & $3 \mathrm{e} 15$ & $3 \mathrm{e} 16$ & $3.0 \mathrm{e} 39$ & 1 & 1 & $3 \mathrm{e} 2$ & $3 \mathrm{e} 4$ & 1.5 & 3.5 & 3.5 & 3.5 \\
Mkn 421 (spine) & $3 \mathrm{e} 15$ & $1 \mathrm{e} 15$ & $3.0 \mathrm{e} 40$ & 1.1 & $2 \mathrm{e} 2$ & $3 \mathrm{e} 4$ & $1 \mathrm{e} 6$ & 1.5 & 3.5 & 17 & 2.5 \\
Mkn 421 (layer) & $3 \mathrm{e} 15$ & $3 \mathrm{e} 16$ & $5.0 \mathrm{e} 39$ & 0.5 & 50 & $1 \mathrm{e} 4$ & $3 \mathrm{e} 5$ & 1.5 & 3.5 & 3 & 2.5 \\
Cen A (spine) & $1 \mathrm{e} 16$ & $1 \mathrm{e} 15$ & $8.0 \mathrm{e} 42$ & 4 & 50 & $3 \mathrm{e} 3$ & $3 \mathrm{e} 4$ & 1.5 & 4 & 15 & 40 \\
Cen A (layer) & $1 \mathrm{e} 16$ & $1 \mathrm{e} 17$ & $1.0 \mathrm{e} 42$ & 2 & $1 \mathrm{e} 2$ & $4 \mathrm{e} 3$ & $4 \mathrm{e} 3$ & 1.0 & 4 & 3 & 40 \\
PKS 0735+178/NGC 6251 (spine) & $5 \mathrm{e} 15$ & $5 \mathrm{e} 14$ & $1.0 \mathrm{e} 42$ & 5 & 50 & $2 \mathrm{e} 3$ & $6 \mathrm{e} 3$ & 1.5 & 3 & 15 & $3 / 20$ \\
PKS 0735+178/NGC 6251 (layer) & $5 \mathrm{e} 15$ & $2.5 \mathrm{e} 16$ & $5.0 \mathrm{e} 41$ & 1.8 & $1 \mathrm{e} 2$ & $2 \mathrm{e} 3$ & $1 \mathrm{e} 4$ & 1.6 & 4.5 & 3 & $3 / 20$ \\
\hline
\end{tabular}

is a potentially important deceleration mechanism, we discuss it here in more detail.

The most convenient frame in which to study the dynamics of the spine is the frame comoving with the layer. In this frame the synchrotron radiation produced by the layer is isotropic, with energy density $U_{\text {syn }}^{\prime \prime}$ To avoid an excess of symbolism, from now on (unless otherwise noted) the unprimed quantities are measured in the frame of the layer, and the primed quantities are evaluated in the frame of the spine. For the same reason we let the random Lorentz factor unprimed, with the notion that these Lorentz factors are measured in the frame of the spine, where they are assumed to be isotropically distributed.

The total Lorentz factor $(\tilde{\gamma})$ of the electrons is the superposition of the relative bulk $(\Gamma)$ and random $(\gamma)$ Lorentz factors. Assume that the random velocity forms an angle $\theta^{\prime}$ with respect to the jet axis, in the spine frame. From Rybicki \& Lightman (1979) we have:

$\beta_{x}=\frac{\beta^{\prime} \cos \theta^{\prime}+\beta_{\text {bulk }}}{1+\beta_{\text {bulk }} \beta^{\prime} \cos \theta^{\prime}}$

$\beta_{y}=\frac{\beta^{\prime} \sin \theta^{\prime}}{\Gamma_{\text {bulk }}\left(1+\beta_{\text {bulk }} \beta^{\prime} \cos \theta^{\prime}\right)}$.

The total $\tilde{\gamma}^{2}$ is

$$
\begin{aligned}
\tilde{\gamma}^{2} & =\left[1-\beta_{x}^{2}-\beta_{y}^{2}\right]^{-1} \\
& =\left(1+\beta_{\text {bulk }} \beta^{\prime} \cos \theta^{\prime}\right)^{2} \gamma^{2} \Gamma^{2} .
\end{aligned}
$$

If the particle distribution is isotropic in the spine frame, the average over all angles gives

$$
\begin{aligned}
\left\langle\tilde{\gamma}^{2}\right\rangle & =\frac{\int 2 \pi \sin \theta^{\prime} \tilde{\gamma}^{2}\left(\theta^{\prime}\right) \mathrm{d} \theta^{\prime}}{4 \pi} \\
& =\frac{\gamma^{2} \Gamma^{2}}{2} \int_{-1}^{1}\left(1+\beta_{\text {bulk }} \beta^{\prime} \mu^{\prime}\right)^{2} \mathrm{~d} \mu^{\prime} \\
& =\left[1+\frac{\left(\beta_{\text {bulk }} \beta^{\prime}\right)^{2}}{3}\right] \gamma^{2} \Gamma^{2}
\end{aligned}
$$

the factor in square parentheses becomes (4/3) for ultrarelativistic speeds.

The loss of energy of the jet is proportional to $\left\langle\tilde{\gamma}^{2}\right\rangle$, and the loss of momentum is the component along the jet axis of the loss of energy. In other words, we have to calculate the quantity

$$
\left\langle\tilde{\gamma}^{2}\right\rangle_{z}=\frac{\int 2 \pi \mu^{\prime} \tilde{\gamma}^{2}\left(\theta^{\prime}\right) \mathrm{d} \mu^{\prime}}{4 \pi}=\frac{2}{3} \beta_{\text {bulk }} \beta^{\prime} \gamma^{2} \Gamma^{2} .
$$

Now assume that the spine carries $N_{\mathrm{p}}$ protons (total number) and $N_{\mathrm{e}}$ leptons. The loss of momentum of the jet is described by:

$$
\begin{aligned}
\frac{\mathrm{d} \Gamma}{\mathrm{d} t} & =\frac{4}{3} \frac{\sigma_{\mathrm{T}} c N_{\mathrm{e}} U_{\text {syn }}\left\langle\tilde{\gamma}^{2}\right\rangle_{z}}{N_{\mathrm{p}} m_{\mathrm{p}} c^{2}+N_{\mathrm{e}}\langle\gamma\rangle m_{\mathrm{e}} c^{2}} \\
& =\frac{8}{9} \frac{\sigma_{\mathrm{T}} c N_{\mathrm{e}} U_{\text {syn }}\left\langle\gamma^{2}\right\rangle \Gamma^{2}}{N_{\mathrm{p}} m_{\mathrm{p}} c^{2}+N_{\mathrm{e}}\langle\gamma\rangle m_{\mathrm{e}} c^{2}} .
\end{aligned}
$$

We recall that the bulk Lorentz in Eq. (10) is the relative Lorentz factor (which is the Lorentz factor of the spine as measured in the layer). A detailed analysis of this Compton rocket effect in AGN jets has been presented by Sikora et al. (1996). They derive the equations regulating the drag effect and apply the results to the case of jets in radio-loud quasars with strong emission lines. Equation (10), although obtained in a rather simplified way, coincides with the results of Sikora et al. (for the specific case of an isotropic radiation field).

To find the value of $\Gamma_{\mathrm{s}}$ as measured by an observer on Earth, one must invert Eq. (1). It must be noted that, in general, $\langle\gamma\rangle$ and $\left\langle\gamma^{2}\right\rangle$ are not constant, but can change due to e.g. radiative cooling. We have integrated Eq. (10) assuming:

- the particle distribution remains unaltered for a time equal to the light crossing time of the spine (i.e. $\Delta R_{\mathrm{s}} / c$ ) as measured in the frame of the layer. This corresponds to assuming that the time of the injection of particles throughout the spine lasts for a similar time;

- after this time we calculate the actual $\gamma_{\text {cool }}$ at the given time, and assume that the particle distribution vanishes for $\gamma>\gamma_{\text {cool }}$. We neglect adiabatic losses, for simplicity;

- we then calculate the new values of $\langle\gamma\rangle$ and $\left\langle\gamma^{2}\right\rangle$ and the new value of $\Gamma$;

- we invert Eq. (1) and finally find $\Gamma_{\mathrm{s}}$.

\section{Results}

\subsection{Fitting Mkn 501, Mkn 421 and Cen A}

We here apply the model to the two best studied TeV BL Lacs: Mkn 501 and Mkn 421, and to the radiogalaxy Cen A.

We stress that our aim is not to precisely fit the SED of these sources, since our results are not unique. For instance, the parameters for the layer of Mkn 421 and Mkn 501 have been chosen with the aim of increasing the allowed value of the 
Table 2. Derived jet powers and parameters of the models for the layer and the spine shown in Figs. 2-5.

\begin{tabular}{llllllllll}
\hline \hline & $\begin{array}{l}L_{\mathrm{e}} \\
\mathrm{erg} \mathrm{s}^{-1}\end{array}$ & $\begin{array}{l}L_{\mathrm{p}} \\
\mathrm{erg} \mathrm{s}^{-1}\end{array}$ & $\begin{array}{l}L_{B} \\
\mathrm{erg} \mathrm{s}^{-1}\end{array}$ & $\begin{array}{l}L_{\mathrm{r}} \\
\mathrm{erg} \mathrm{s}^{-1}\end{array}$ & $\begin{array}{l}L_{\mathrm{s}} \\
\mathrm{erg} \mathrm{s}^{-1}\end{array}$ & $\langle\gamma\rangle$ & $\left\langle\gamma^{2}\right\rangle$ & $\Gamma^{\prime}$ & $\delta$ \\
\hline Mkn 501 (spine) & $3.7 \mathrm{e} 42$ & $6.7 \mathrm{e} 43$ & $1.3 \mathrm{e} 43$ & $6.1 \mathrm{e} 43$ & $5.8 \mathrm{e} 43$ & 102 & $2.1 \mathrm{e} 7$ & 2.3 & 16.3 \\
Mkn 501 (layer) & $1.3 \mathrm{e} 41$ & $1.7 \mathrm{e} 43$ & $1.7 \mathrm{e} 41$ & $5.9 \mathrm{e} 40$ & $3.9 \mathrm{e} 39$ & 14 & $2.9 \mathrm{e} 3$ & 2.3 & 6.6 \\
Mkn 421 (spine) & $1.0 \mathrm{e} 43$ & $9.7 \mathrm{e} 42$ & $1.2 \mathrm{e} 43$ & $1.3 \mathrm{e} 43$ & $8.9 \mathrm{e} 42$ & $1.9 \mathrm{e} 3$ & $3.1 \mathrm{e} 7$ & 3.0 & 21.9 \\
Mkn 421 (layer) & $2.5 \mathrm{e} 40$ & $8.5 \mathrm{e} 40$ & $3.2 \mathrm{e} 40$ & $1.2 \mathrm{e} 41$ & $5.6 \mathrm{e} 39$ & $5.4 \mathrm{e} 2$ & $2.8 \mathrm{e} 6$ & 3.0 & 5.7 \\
Cen A (spine) & $2.7 \mathrm{e} 45$ & $1.9 \mathrm{e} 46$ & $1.3 \mathrm{e} 45$ & $4.6 \mathrm{e} 45$ & $1.8 \mathrm{e} 45$ & $2.6 \mathrm{e} 2$ & $2.5 \mathrm{e} 5$ & 2.7 & 0.28 \\
Cen A (layer) & $7.7 \mathrm{e} 41$ & $4.1 \mathrm{e} 42$ & $2.7 \mathrm{e} 41$ & $5.1 \mathrm{e} 43$ & $9.6 \mathrm{e} 41$ & $3.5 \mathrm{e} 2$ & $2.2 \mathrm{e} 5$ & 2.7 & 1.2 \\
PKS 0735+178/NGC 6251 (spine) & $8.0 \mathrm{e} 44$ & $7.4 \mathrm{e} 45$ & $5.3 \mathrm{e} 44$ & $4.0 \mathrm{e} 44$ & $2.2 \mathrm{e} 44$ & 198 & $1 \mathrm{e} 5$ & 2.7 & 18.6 \\
PKS 0735+178/NGC 6251 (layer) & $2.3 \mathrm{e} 42$ & $1.6 \mathrm{e} 43$ & $5.4 \mathrm{e} 41$ & $3.9 \mathrm{e} 43$ & $9.4 \mathrm{e} 41$ & 271 & $1.4 \mathrm{e} 5$ & 2.7 & 2.9 \\
\hline
\end{tabular}

magnetic field of the corresponding spines. In other words, we applied a theoretical prejudice, aiming to bring these sources closer to equipartition than a simple one zone SSC model allows us to do. Other poorly constrained parameters are the width of the layer and its bulk Lorentz factor. For the former we simply require $\Delta R^{\prime \prime}>R$, for the latter we have the requirement that the counterjet is, in these sources, invisible up to the few tens of m.a.s. scale, leading to a limit of $\Gamma_{1}>2-3$.

In conclusion, our aim is to demonstrate that our model can consistently work both for radiogalaxies and BL Lacs, and this makes it possible to draw some general conclusions about their jets and the importance of the inverse Compton emission. The input parameters used for the models shown in Figs. 2-5 are all listed in Table 1, while Table 2 reports the values of the kinetic powers carried by the spine and the layer, the average (and the average of the square) of the random Lorentz factor of the emitting electrons, and the relative bulk Lorentz factor $\Gamma^{\prime}$. The kinetic powers are defined as

$L_{i}=\pi R^{2} \Gamma^{2} \beta c U_{i}$

where the energy density $U_{i}$ refers to: electrons $\left(U_{\mathrm{e}}=\right.$ $\left.\int N(\gamma) \gamma m_{\mathrm{e}} c^{2} \mathrm{~d} \gamma\right)$; magnetic field $\left(U_{B}=B^{2} / 8 \pi\right)$; protons $\left(U_{\mathrm{p}}\right)$, assuming one cold proton per electron; total radiation $\left(U_{\mathrm{rad}}\right)$ and synchrotron radiation $\left(U_{\text {syn }}\right)$.

In all cases shown in Figs. 2-5, continuous lines show the results of the models including the spine-layer radiative feedback, while the dashed lines are the results of the same models neglecting the feedback.

- Mkn 501: Fig. 2 shows the "fits" to the SED of Mkn 501 in its flaring state of April 1997 (Pian et al. 1998). Note the rather moderate bulk Lorentz factor used for the spine $\left(\Gamma_{\mathrm{s}}=15\right)$, which has the same value as the one used by GK03. The rather large value of the magnetic field ensures near-equipartition between magnetic and particle energy

- Mkn 421: Figure 3 shows our model compared with different simultaneous SED of this source, and aiming to "fit" the highest state. The bulk Lorentz factor of the spine is $\Gamma_{\mathrm{s}}=$ 17 , which at a viewing angle $\theta=2.5^{\circ}$ leads to a beaming factor $\delta=22$. The remarkable difference with respect to a simple one zone SSC model is the much larger value of the magnetic field: 1 Gauss compared with $B \sim 0.02-0.1$ Gauss (see the references quoted in the Introduction). This brings the magnetic energy into equipartition with the particle energy, dominated in this case by the energetic electrons, which have a very large average random Lorentz factor $\left(\langle\gamma\rangle=1900\right.$, and therefore $\left.\langle\gamma\rangle m_{\mathrm{e}} \sim m_{\mathrm{p}}\right)$. This is due both to the relatively large value of $\gamma_{\min }$ and the flat electron slope at low energies, in turn required in order not to overproduce the IR emission.

- Cen A: as can be seen in Fig. 3, the synchrotron spectral component in this object is particularly narrow, with a peak in the far IR. This indicates a correspondingly narrow energy distribution of the emitting electrons. We have assumed a viewing angle of $\theta=40^{\circ}$, corresponding to a modest beaming of the layer emission $(\delta=1.2)$, and a severe de-beaming of the spine radiation $(\delta=0.28)$. Comparing the jet powers of this source with Mkn 421 or Mkn 501, we find that the jet of Cen $\mathrm{A}$ is more powerful, with most of the power carried by the spine.

\section{2. "Pairing" BL Lacs with radiogalaxies}

We here try to see if our spine+layer structure can explain at the same time the emission from a "classical" BL Lac object, PKS $0735+178$, and the radiation observed for the radiogalaxy NGC 6251, which has recently been associated with an EGRET source. In other words, we check if these two apparently very different objects, at very different distances (but both emitting high energy $\gamma$-rays), can be considered as "paired". Note that the radio luminosity $L_{1.4}$ at $1.4 \mathrm{GHz}$ of the two objects, indicative of the extended (and unbeamed) radio power, is almost equal (for PKS 0735+178: $L_{1.4}=9 \times 10^{31} \mathrm{erg} \mathrm{s}^{-1} \mathrm{~Hz}^{-1}$, Cassaro et al. 1999; for NGC 6251: $L_{1.4}=3 \times 10^{31} \mathrm{erg} \mathrm{s}^{-1} \mathrm{~Hz}^{-1}$, Laing et al. 1983, extrapolating from $178 \mathrm{MHz}$ with a radio spectral index $\alpha=0.7)$. In this case the differences in the observed nuclear SED are due entirely to a different viewing angle $\theta$, enhancing the spine or the layer emission for small and large $\theta$, respectively. As a consequence, we now have much less freedom than before for the fitting, but, of course, now it is the choice of these two sources which is somewhat arbitrary, even if both are $\gamma$-ray emitters and presumably belong to the FR I class of radiogalaxies.

- PKS 0735+178: this "classic" BL Lac has been detected by EGRET (Hartman et al. 1999), and shows a relatively 


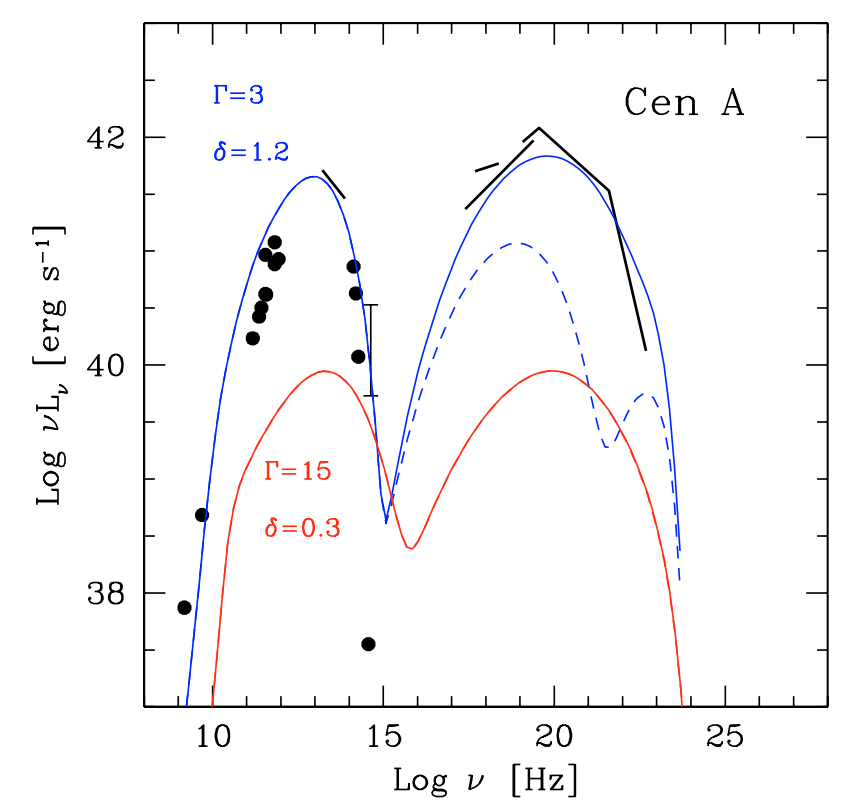

Fig. 3. The SED of Cen A is modelled by the spine-layer system, using the parameters listed in Table 1. Dashed lines correspond to the emission of the spine (layer) without taking into account the seed photons coming from the layer (spine). For the spine the dashed and continuous lines overlap. Data from Chiaberge et al. (2001) and references therein.

flat X-ray spectrum, signature of the fact that at these energies the inverse Compton emission is already dominating. The synchrotron peak frequency lies in the IR-optical and possibly "moves" within these bands. The data shown in Fig. 4 are not simultaneous, and therefore give a rough idea of the entire SED. Nevertheless it is clear that this source belongs to the LBL (Low energy peak BL Lac, see Giommi \& Padovani 1995) category. There is only a lower limit for its redshift, $(z>0.424)$; for the modeling we assume $z=0.424$. The implied energetics are two orders of magnitude larger than for the previous sources, if a similar value of the Doppler beaming factor is appropriate. Accordingly, we have assumed a larger intrinsic power, and a larger value of the magnetic field. This brings the magnetic energy closer to equipartition (with respect to a pure SSC model) with the energy contained in the relativistic electrons.

- NGC 6251: the layer assumed to be in the jet of PKS $0735+178$, if observed at $20^{\circ}$, becomes the dominant contributor to the SED and gives rise to the spectrum of the nearby radiogalaxy NGC $6251(z=0.0249)$, as illustrated in the bottom panel of Fig. 4. Note that also in this case the data points are not simultaneous, and can only give a rough idea of the overall (nuclear) SED of this object. The beamed non-thermal component, being variable with a relatively large amplitude, could dominate the total flux only occasionaly, letting in other cases the component produced by the accretion disk be dominant. This could also explain why, in some observations, a relatively strong fluorescent broad iron line (at $6.4 \mathrm{keV}$ ) is visible (Gliozzi et al. 2004). Bearing in mind these uncertainties in the relative strength

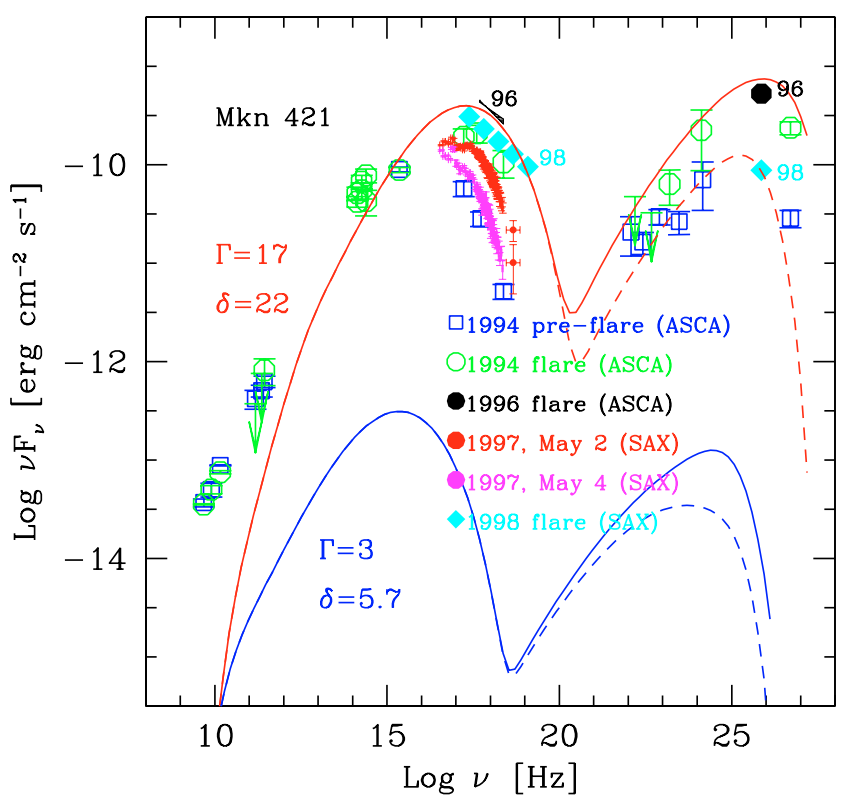

Fig. 4. Example of the SED produced by the spine-layer system, using the parameters listed in Table 1. Dashed lines correspond to the emission of the spine (layer) without taking into account the seed photons coming from the layer (spine). For the data points see Costamante \& Ghisellini (2002) and references therein.

of the beamed vs accretion components, the slight overproduction of X-rays predicted by the model is not (yet) necessarily a failure of the model. Note also that when the layer is not illuminated by the spine (and this can happen, since the spine can be constituted by discontinuous blobs), then the predicted emission corresponds to the dashed line, which is closer to the X-ray data, but underpredicts the $\gamma$-ray flux. This may originate from two different states of the source, and EGRET could detect the object only when the layer was illuminated by the spine. Note that this source showed low amplitude, relatively fast $\left(\sim 10^{4} \mathrm{~s}\right)$ variability in hard $\mathrm{X}$-rays (above $0.8 \mathrm{keV}$ ), in agreement with the idea that the non-thermal beamed continuum rivals the contribution produced by the accretion disk, with the latter better visible in the softer X-ray band (Gliozzi et al. 2004).

\subsection{Jet deceleration by the Compton rocket effect}

As anticipated above, from Table 2 we can infer that the power released as inverse Compton radiation by the spines of Mkn 501 and Mkn 421 is comparable to the total kinetic power carried by the jet. In these circumstances the Compton rocket effect is important for determining the dynamics. For illustration, Fig. 6 shows the evolution of the bulk Lorentz factor in the case of Mkn 421, calculated along the lines discussed in Sect. 3, and assuming the same parameters as used for the fitting described above and listed in Table 1. We show the bulk Lorentz factor as measured by the layer and also by the observer at earth, but both as a function of the distance as measured in the frame of the layer. We also show the logarithm of $\gamma_{\text {cool }}$. As can be seen, the recoil is very significant, decelerating the spine from $\Gamma_{\mathrm{s}}=17$ to $\Gamma_{\mathrm{s}}=7.5$ within the layer. 


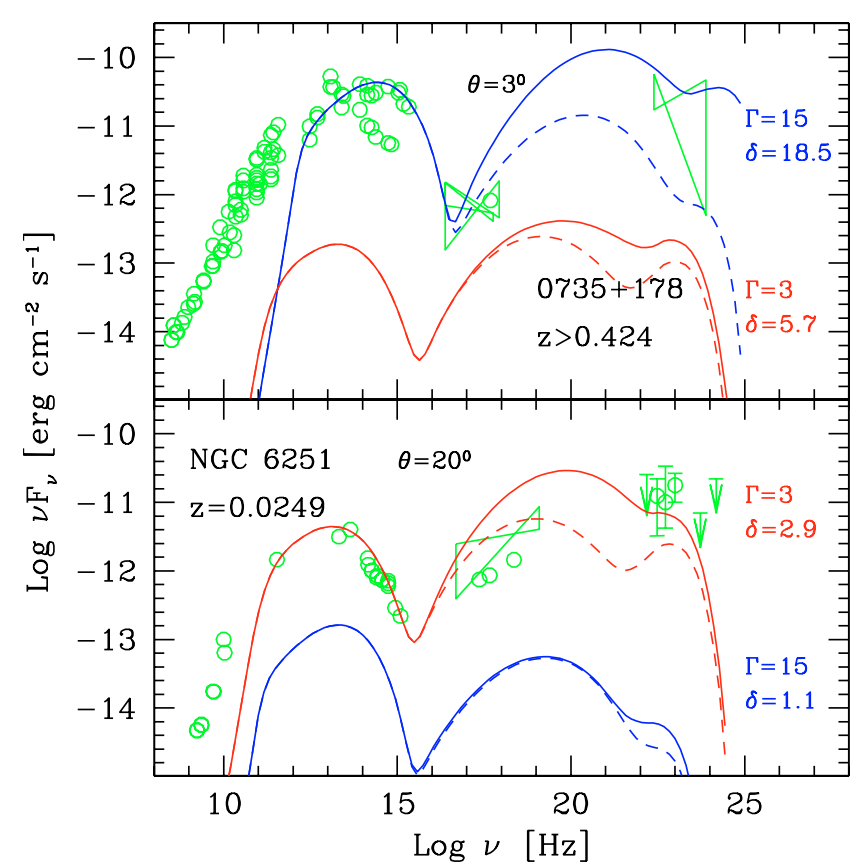

Fig. 5. Example of the SED produced by the spine-layer system, using the parameters listed in Table 1 . The viewing angle is $\theta=2^{\circ}$ for the example shown in the upper panel, and $\theta=22^{\circ}$ for the bottom panel. The model is compared with the data of the BL Lac PKS 0735+178 (top) and the FR 1 radiogalaxy NGC 6251 (bottom). Dashed lines correspond to the emission of the spine (layer) without taking into account the seed photons coming from the layer (spine). Data from Ghisellini et al. (1998) and Chiaberge et al. (2003) and references therein.

Note that $\Gamma=3$ is the bulk Lorentz factor of the layer, which is therefore the minimum value which the spine could attain.

\subsection{General outcomes and implications}

From the exercise of "fitting" the SED of the previous few BL Lacs and radiogalaxies, we can draw the following general conclusions:

- The feedback between the layer and the spine enhances the inverse Compton emission in both components. In the examples shown, the enhancement is around one order of magnitude, or even greater.

- The magnetic field is larger, in this spine/layer scenario, and is consistent with being in equipartition with the relativistic electrons.

- The larger magnetic field implies that, to produce the same amount of synchrotron radiation, fewer electrons are needed. If there is one proton per electron, we also need fewer protons. This implies a decrease in the total jet kinetic power (with respect to a pure SSC model).

- The Compton rocket effect can influence the dynamics of the spine, since the external Compton luminosity (being anisotropic in the comoving frame) becomes comparable with the total kinetic power carried by the spine itself.

- The examples shown here assume that the spine is active only when inside the layer. But other possibilities exist: for instance, the layer could be particularly narrow (i.e.

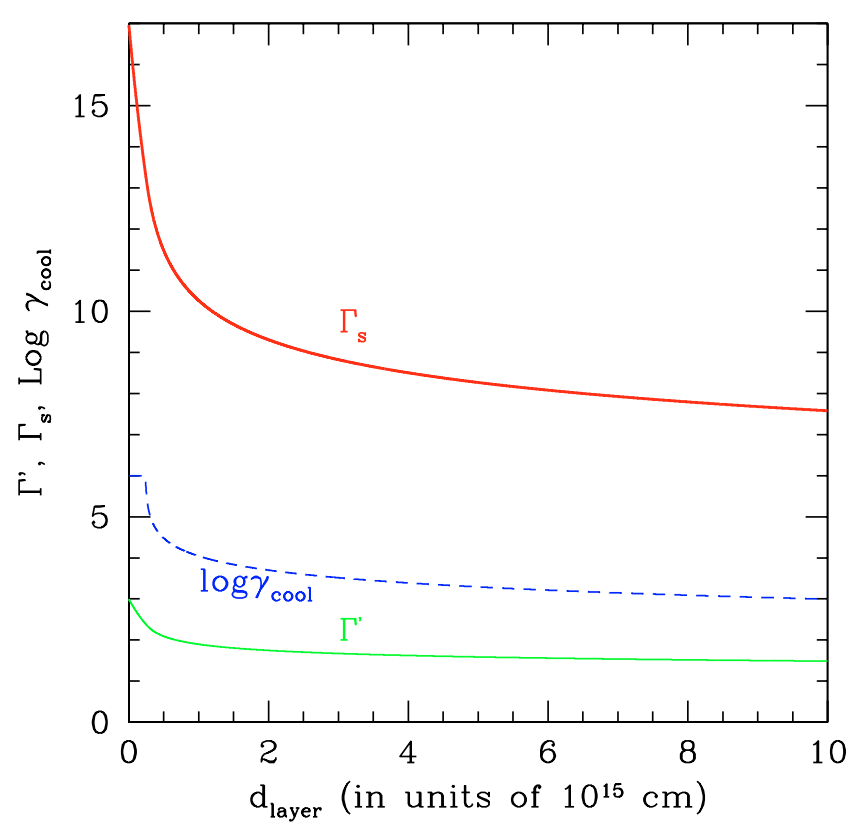

Fig. 6. The bulk Lorentz factor as a function of distance as measured in the frame of the layer and in the Earth frame. Also shown, as labelled, is the logarithm of $\gamma_{\mathrm{cool}}$. We have adopted the same parameters as the ones used for Mkn 421 and reported in Table 1.

small $\Delta R_{1}$ ), so that the spine would be able to survive when passing it (i.e. the electrons may not cool completely). In such a case, the spine will continue to emit the same amount of synchrotron radiation (if the particle distribution does not change), while the amount of high energy emission will decrease drastically. This might explain the "orphan $\mathrm{TeV}$ flares" (i.e. flares in the TeV band not accompanied by simultaneous flares in the X-ray band) observed in simultaneous RXTE/TeV observations (Krawczynski et al. 2004).

- To be effective, the layer must of course be located in the region of the jet where the spine undergoes dissipation and emits. In other words, it must be compact, even if its width can be larger (factor $\sim 10$ ) than the spine width (i.e. $\left.\Delta R_{\mathrm{l}}{ }^{\prime \prime} \sim 10 \Delta R_{\mathrm{s}}^{\prime}\right)$. This implies variability of the layer. Even if its particle distribution is steady, its high energy emission will change depending on if the spine is illuminating it or not (e.g. compare the continuous and dashed lines for the layer in Figs. 2-5).

\section{5. $\mathrm{GeV}$ and $\mathrm{TeV}$ radiogalaxies?}

The slow layer also produces a large amount of $\mathrm{GeV}$ radiation, which remains visible even at large viewing angles (consider that $\theta=1 / \Gamma_{\text {layer }} \sim 20^{\circ}$ ). Therefore also radiogalaxies should be high energy emitters. Indeed, the three radiogalaxies detected at high energies so far, shown in Fig. 7, show the characteristic double bump SED typical of blazars, suggesting a similar origin of their emission. This is clearer in Fig. 8, which compares directly the averaged SED of blazars with the SED of Cen A, NGC 6251 and M 87.

For Cen A and NGC 6251, which have been identified with EGRET surces, we measure ratios between the EGRET $\left(v F_{v}\right)$ flux at $100 \mathrm{MeV}$ and the radio flux of the core at $5 \mathrm{GHz}$ which is 


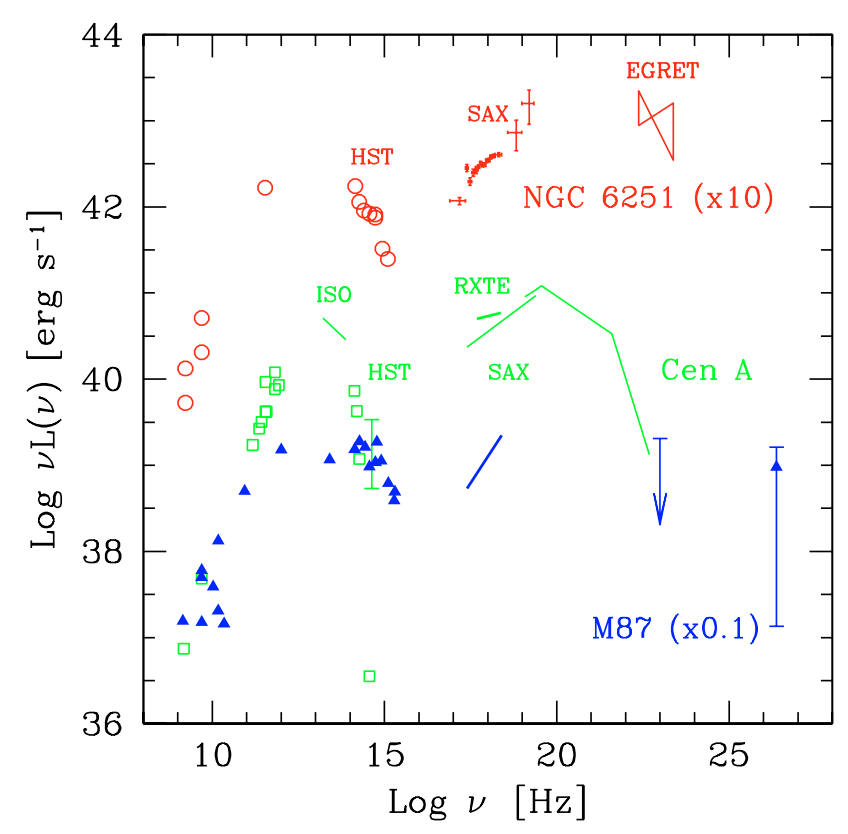

Fig. 7. The SED of NGC 6251, Cen A and M 87. The data of NGC 6251 and M 87 have been vertically shifted by the labelled amount for clarity. Note that the SED of these radiogalaxies show the two-bump structure of blazars, as illustrated also in Fig. 8. Data for M 87 are taken from Reimer et al. (2004) and references therein, except for the X-rays (Marshall et al. 2002), and the EGRET upper limit (Fichtel et al. 1994).

of 60 and 300, respectively. Such a large ratio is also consistent with the $\mathrm{TeV}$ detection of M 87, if the peak of its $\gamma$-ray emission lies between the EGRET and the TeV band.

If all FR I radiogalaxies have a similar $\gamma$-ray to radio flux ratio, we can identify the best candidates for detection in future AGILE and GLAST observations. To this aim, we have averaged the ratio of the $\gamma$-ray to radio flux for the three radiogalaxies detected in $\gamma$-rays which results in

$\log v_{\gamma} F_{\gamma}=(2 \pm 0.5)+\log v_{\mathrm{R}} F_{\mathrm{R}, \text { core }}$

where $F_{\mathrm{R} \text {,core }}$ is the radio flux of the core at $5 \mathrm{GHz}$ (Giovannini et al. 1998; Chiaberge et al. 1999 and references therein), and $F_{\gamma}$ is the $\gamma$-ray flux at $100 \mathrm{MeV}$. Using Eq. (12) as an empirical guide to predict the $\gamma$-ray fluxes of radiogalaxies, we list in Table 3 the FR I radiogalaxies belonging to the $3 \mathrm{C}$ sample sample, in order of decreasing nuclear radio power, and the corresponding predicted $100 \mathrm{MeV}$ flux. Assuming a sensitivity limit of GLAST of $\sim 5 \times 10^{-13} \mathrm{erg} \mathrm{cm}^{-2} \mathrm{~s}^{-1}$ above $100 \mathrm{MeV}$ for one year of exposure time, i.e. a factor 20 better than EGRET, (see e.g.: http://glast.gsfc.nasa.gov), we expect that more than a dozen FR I radiogalaxies can be detected.

\section{Discussion}

We have explored the radiative and dynamical consequences of a structured jet in which a slow jet layer is cospatial to a fast jet spine. The motivations for this study are primarily observational, since radio data have recently found slow or null proper motion for the parsec scale radio knots in $\mathrm{TeV}$-emitting

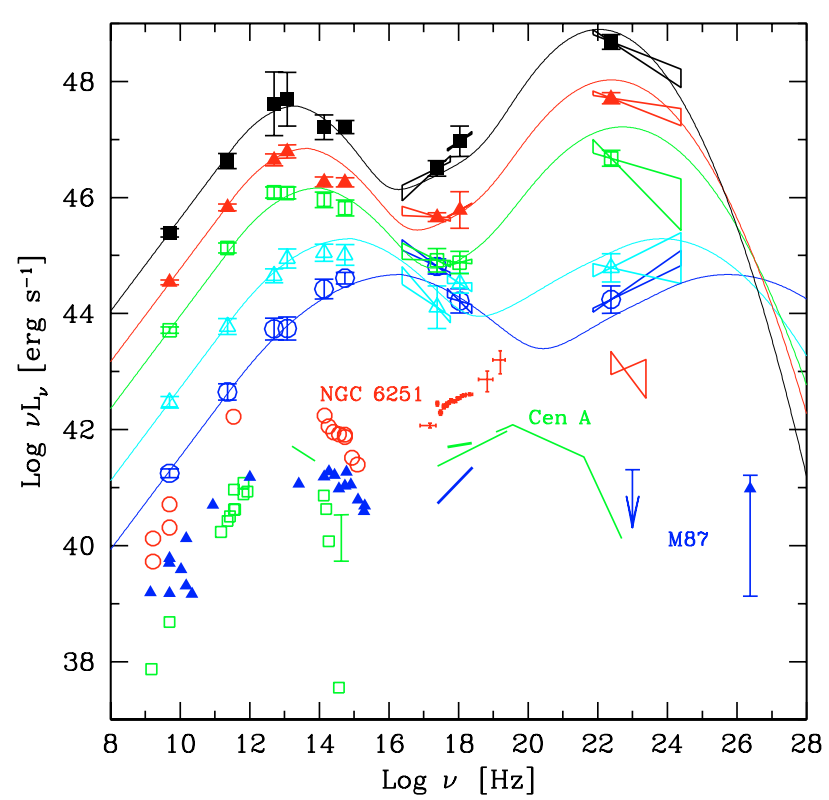

Fig. 8. The SEDs of NGC 6251, Cen A and M 87 are compared with the blazar sequence, as proposed by Fossati et al. (1998). The hard X-ray [2-10 keV] spectra of blazars come from the work of Donato et al. (2001).

Table 3. Radio core fluxes at $5 \mathrm{GHz}$ for $3 \mathrm{CR}$ FR I and predicted gamma-ray fluxes. See the text for the assumptions.

\begin{tabular}{|c|c|c|}
\hline Name & $\begin{array}{c}\log F_{\mathrm{R}, \text { core }} \\
(5 \mathrm{GHz}) \\
\mathrm{erg} \mathrm{cm}^{-2} \mathrm{~s}^{-1} \mathrm{~Hz}^{-1}\end{array}$ & $\begin{array}{c}\log v_{\gamma} F_{\gamma} \\
(100 \mathrm{MeV}) \\
\operatorname{erg~cm}^{-2} \mathrm{~s}^{-1}\end{array}$ \\
\hline $3 \mathrm{C} 84$ & -21.37 & $-9.67 \pm 0.5$ \\
\hline $3 \mathrm{C} 274$ & -22.40 & $-10.70 \pm 0.5$ \\
\hline 3C 78 & -23.02 & $-11.32 \pm 0.5$ \\
\hline $3 \mathrm{C} 317$ & -23.41 & $-11.71 \pm 0.5$ \\
\hline $3 \mathrm{C} 270$ & -23.51 & $-11.81 \pm 0.5$ \\
\hline $3 \mathrm{C} 465$ & -23.57 & $-11.87 \pm 0.5$ \\
\hline $3 \mathrm{C} 346$ & -23.66 & $-11.96 \pm 0.5$ \\
\hline $3 \mathrm{C} 264$ & -23.70 & $-12.00 \pm 0.5$ \\
\hline $3 \mathrm{C} 66$ & -23.74 & $-12.04 \pm 0.5$ \\
\hline 3C 272.1 & -23.74 & $-12.05 \pm 0.5$ \\
\hline $3 \mathrm{C} 315$ & -23.82 & $-12.12 \pm 0.5$ \\
\hline 3C 338 & -23.98 & $-12.28 \pm 0.5$ \\
\hline 3C 293 & -24.00 & $-12.30 \pm 0.5$ \\
\hline $3 \mathrm{C} 29$ & -24.03 & $-12.33 \pm 0.5$ \\
\hline $3 C 31$ & -24.04 & $-12.34 \pm 0.5$ \\
\hline 3C 310 & -24.10 & $-12.40 \pm 0.5$ \\
\hline 3C 296 & -24.11 & $-12.41 \pm 0.5$ \\
\hline $3 C 89$ & -24.31 & $-12.61 \pm 0.5$ \\
\hline $3 \mathrm{C} 75$ & -24.41 & $-12.71 \pm 0.5$ \\
\hline $3 \mathrm{C} 449$ & -24.43 & $-12.73 \pm 0.5$ \\
\hline $3 \mathrm{C} 288$ & -24.52 & $-12.82 \pm 0.5$ \\
\hline $3 \mathrm{C} 305$ & -24.53 & $-12.83 \pm 0.5$ \\
\hline 3C 83.1 & -24.68 & $-12.98 \pm 0.5$ \\
\hline $3 \mathrm{C} 424$ & -24.74 & $-13.05 \pm 0.5$ \\
\hline $3 \mathrm{C} 438$ & -24.77 & $-13.07 \pm 0.5$ \\
\hline $3 \mathrm{C} 386$ & -24.77 & $-13.07 \pm 0.5$ \\
\hline $3 \mathrm{C} 277.3$ & -24.91 & $-13.21 \pm 0.5$ \\
\hline 3C 348 & -25.00 & $-13.30 \pm 0.5$ \\
\hline $3 \mathrm{C} 433$ & -25.30 & $-13.60 \pm 0.5$ \\
\hline $3 \mathrm{C} 442$ & -25.70 & $-14.00 \pm 0.5$ \\
\hline
\end{tabular}


BL Lacs. In addition, detailed VLBI radio maps show hints of a limb brightening for the jet in Mkn 501.

The jet may be born as structured, or it may be born with equal layer/spine velocities, with the layer being decelerated in the first $\sim 10^{17} \mathrm{~cm}$ from the central black hole. As long as the layer is dissipative, our results are independent of the mechanism producing a spine+layer structure. However, we note that the accretion disk in low power BL Lacs could be characterized by a low-efficiency accretion mode, and one of the proposed accretion disk solutions in this case is the ADIOS structure (Blandford \& Begelman 1999), which predicts that a sizeable fraction of the accreting mass at large radii, instead of spiralling inward, leaves the disk in the form of an outflow. In this case, in the close vicinity of the black hole the ambient medium should be relatively dense, favoring the interaction of the jet walls with the external medium. This may cause the formation of the slow layer, and at the same time this interaction may be the primary cause for the transformation of the kinetic energy of the layer into random energy and then radiation.

A low-radiative efficiency accretion disk could also explain why the broad line emission is low in BL Lac objects and in FR I radiogalaxies. Further evidence of a change in the accretion mode between FR I and FR II radiogalaxies is provided by the "dividing line" between these two classes of sources in the radio-luminosity-host galaxy optical luminosity plane, as discussed by Ghisellini \& Celotti (2001).

If indeed the jet forms (or is born with) a layer+spine structure, then there is a radiative interplay or feedback between the two parts: each component would see an enhanced radiation field coming from the other component. This would inevitably boost the inverse Compton radiation with respect to a completely homogeneous jet.

It is also quite clear that, for an observed Compton to synchrotron power ratio, the fact that the radiation field is enhanced also implies an increase of the magnetic field, with respect to a homogeneous source. This can solve an otherwise puzzling characteristic of HBL in general and TeV BL Lacs in particular: fitted with a homogeneous SSC model, they turn out to have very small magnetic fields, which are more underequipartition (with the emitting particles) than in other blazars. Here we can fit the observed spectra equally well (not surprisingly, given that there are more free parameters than in the homogeneous SSC model) with equipartition magnetic fields.

Another important consequence of having stronger magnetic fields is that a smaller number of electrons can produce the observed SED. This means that the global energetic demand of the jet is reduced with respect to a homogeneous SSC model.

The fact that the dominant inverse Compton radiation arises through scattering with "external" photons implies that the emission is highly anisotropic also in the comoving frame of the spine. To conserve momentum, the emitting spine must recoil and therefore decelerate. This is a manifestation of the "Compton rocket" effect, studied in the early eighties (see e.g. O'Dell 1981) as the means to radiatively accelerate jets. Somewhat ironically, we have shown here that this process can be important for the opposite reason. There is a precise link between $\mathrm{TeV}$ emission and deceleration, for two reasons: i) $\mathrm{TeV}$ emitting BL Lacs have the least powerful jets, and yet they move with bulk Lorentz factor equal to or greater than those of other blazars; ii) to produce significant $\mathrm{TeV}$ radiation, the mean energy of the emitting electrons must be large. Then in these sources we have $\langle\gamma\rangle m_{\mathrm{e}} \sim m_{\mathrm{p}}$ : the power carried by the jet in the form of protons and electrons is similar. We then propose that the jet deceleration at small (sub-pc) scales is more efficient in low-power jets emitting high energy radiation. Consider also that the initially fastest sources are the ones suffering the strongest Compton rocket effect.

Also the inverse Compton emission from the layer is enhanced by the extra seed photons coming from the spine. This could be the reason why radiogalaxies are relatively strong $\gamma$-ray emitters. Then the layer and the spine must then be cospatial, and therefore the $\gamma$-ray emitting layer must be located at $\sim 100$ Schwarzschild radii, as the spine. The $\gamma$-ray flux observed in radiogalaxies should then be variable, with timescales of the order of $t_{\mathrm{var}} \sim(R / c) / \delta_{1} \sim$ a day or less, very similar (albeit somewhat longer, because of the smaller Doppler factor) to the typical variability timescale in blazars. We propose this as a crucial test for our scenario. Note that Cen A is already known to vary with short timescales (0.5-4 days) in the $\gamma$-ray band (Kinzer et al. 1995; Steinle et al. 1998).

A few radiogalaxies have already been detected at high energies. The SED of their nuclear emission shows the characteristic double peak characteristic of blazars, and like these sources they can be "Compton dominated" (namely, the high energy component is more luminous than the synchrotron component). These general features are quite easily explained in the layer+spine scenario. We have then tried to predict which are the best candidate radiogalaxies to be detected by the future GLAST mission, assuming a twenty-fold increase in sensitivity with respect to EGRET. As a result, more than a dozen FR I radiogalaxies should be detectable by GLAST, if their radio to $100 \mathrm{MeV}$ flux ratio is similar to that of the three objects already identified by EGRET and by TeV Cherenkov telescopes.

\section{References}

Aharonian, F., Akhperjanian, A., Beilicke, M., et al. 2003, A\&A, 403 L1

Blandford, R. D., \& Begelman, M. C. 1999, MNRAS, 303, L1

Bodo, G., Rossi, P., Mignone, A., Massaglia, S., \& Ferrari, A. 2003, NewAR, 47, 557

Cassaro, P., Stanghellini, C., Bondi, M., et al. 1999, A\&AS, 139, 601

Chiaberge, M., Capetti, A., \& Celotti, A. 1999, A\&A, 349, 77

Chiaberge, M., Celotti, A., Capetti, A., \& Ghisellini, G. 2000, A\&A, 358,104

Chiaberge, M., Gilli, R., Capetti, A., \& Macchetto, F. D. 2003, ApJ, 97, 166

Chiaberge, M., Capetti, A., \& Celotti, A. 2001, MNRAS, 324, L33

Costamante, L., \& Ghisellini, G. 2002, A\&A, 384, 56

Dermer, C. D. 1995, ApJ, 446, L63

Djannati-Atai, A., Piron, F., Barrau, A., et al. A\&A, 350, 17

Donato, D., Ghisellini, G., Tagliaferri, G., \& Fossati, G. 2001, A\&A, 375,739

Dondi, L., \& Ghisellini, G. 1995, MNRAS, 273, 583

Edwards, P. G., \& Piner, B. G. 2002, ApJ, 579, L70

Ferrari, A. 1998, ARA\&A, 36, 539

Fichtel, C. E., Bertsch, D. L., Chiang, J., et al. 1994, ApJS, 94, 551 
Fossati, G., Maraschi, L., Celotti, A., Comastri, A., \& Ghisellini, G. 1998, MNRAS, 299, 433

Georganopoulos, M., \& Kazanas, D. 2003, ApJ, 594, L27 (GK03)

Ghisellini, G. 1999, Astron. Nachrichten, 320, 232

Ghisellini, G., \& Celotti, A. 2001, A\&A, 379, L1

Ghisellini, G., Celotti, A., Fossati, G., Maraschi, L., \& Comastri, A. 1998, MNRAS, 301, 451

Ghisellini, G., Celotti, A., \& Costamante, L. 2002, A\&A, 386, 833

Giommi, P., \& Padovani, P. 1995, MNRAS, 277, 1477

Giovannini, G., Feretti, L., Gregorini, L., \& Parma, P. 1988, A\&A, 199,73

Giovannini, G., Taylor, G. B., Arbizzani, E., et al. 1999, ApJ, 522, 101

Giroletti, M., Giovannini, G., Feretti, L., et al. 2004, ApJ, 600, 127

Gliozzi, M., Sambruna, R. M., Brandt, W. N., Mushotzky, R., \& Eracleous, M. 2004, A\&A, 413, 139

Guetta, D., Ghisellini, G., Lazzati, D., \& Celotti, A. 2004, A\&A, 421, 877

Hartman, R. C., Bertsch, S. D., Bloom, S. D., et al. 1999, ApJS, 123, 79

Henri, G., \& Pelletier, G. 1991, ApJ, 383, L7

Katarzynski, K., Sol, H., \& Kus, A. 2003, A\&A, 410, 101

Kino, M., Takahara, F., \& Kusunose, M. 2002, ApJ, 564, 97

Kinzer, R. L., Johnson, W. N., Dermer, C. D., et al. ApJ, 449, 105

Komissarov, S. S. 1990, SvA Lett., 16, 284

Konopelko, K., Mastichiadis, A., Kirk, J., De Jager, O. C., \& Stecker, F. W. 2003, ApJ, 597, 851

Krawczynski, H., Coppi, P. S., \& Aharonian, F. 2002, MNRAS, 336, 721

Krawczynski, H., Hughes, S. B., Horan, D., et al. 2004, ApJ, 601, 151
Laing, R. A. 1993, in Astrophysical jets, Space Telescope Sci. Inst. Symp., 6, ed. D. Burgarella, M. Livio, \& C. P. O’Dea (Cambridge Univ. Press), 95

Laing, R. A., Riley, J. M., \& Longair, M. S. 1983, MNRAS, 204, 151

Morganti, R., Fosbury, R. A. E., Hook, R. N., Robinson, A., \& Tsvetanov, Z. 1992, MNRAS, 256, P1

Marshall, H. L., Miller, B. P., Davis, D. S., et al. 2002, ApJ, 564, 683

Mukherjee, R., Halpern, J., Mirabal, N., \& Gotthelf, E. V. 2002, ApJ, 574,693

O’Dell, S. L. 1981, ApJ, 243, L147

Owen, F. N., Hardee, P. E., \& Cornwell, T. J. 1989, ApJ, 340, 698

Pian, E., Vacanti, G., Tagliaferri, G., et al. 1998, ApJ, 492, L17

Piner, B. G., \& Edwards, P. G. 2004, ApJ, 600, 115

Rawlings, S. G., \& Saunders, R. D. E. 1991, Nature, 349, 138

Reimer, A., Protheroe, R. J., \& Donea, A.-C. 2004, A\&A, 419, 89

Rybicki, G. B., \& Lightman, A. P. 1979, Radiative Processes in Astrophysics (New York: Wiley Interscience)

Rossi, E. M., Lazzati, D., \& Rees, M. J. 2002, MNRAS, 332, 945

Sikora, M., Sol, H., Begelman, M. C., \& Madejski, G. M. 1996, MNRAS, 280, 781

Spada, M., Ghisellini, G., Lazzati, D., \& Celotti, A. 2001, MNRAS, 325,1559

Stawarz, L., Sikora, M., \& Ostrowski, M. 2003, ApJ, 597, 186

Steinle, H., Bennet, K., Bloemen, H., et al. 1998, A\&A, 330, 97

Swain, M. R., Bridle, A. H., \& Baum, S. A. 1998, ApJ, 507, L29

Tavecchio, F., Maraschi, L., \& Ghisellini, G. 1998, ApJ, 509, 608

Tavecchio, F., Maraschi, L., Pian, E., et al. 2001, ApJ, 554, 725

Zhang, B., \& Meszaros, P. 2002, ApJ, 571, 876 